Influence of hydrodynamic coupling on the electric linear dichroism of DNA fragments

Juan P. Umazano, and Jorge A. Bertolotto

Citation: The Journal of Chemical Physics 134, 125107 (2011); doi: 10.1063/1.3568270

View online: https://doi.org/10.1063/1.3568270

View Table of Contents: http://aip.scitation.org/toc/jcp/134/12

Published by the American Institute of Physics

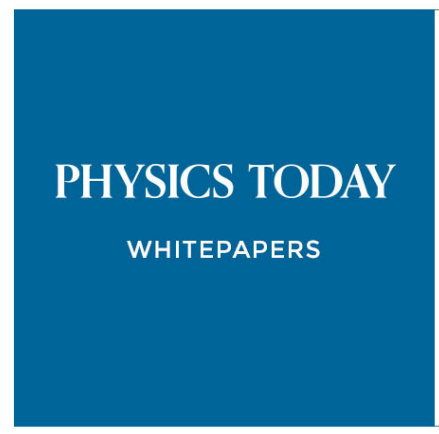
systems can do 


\title{
Influence of hydrodynamic coupling on the electric linear dichroism of DNA fragments
}

\author{
Juan P. Umazano ${ }^{1,2,3, a)}$ and Jorge A. Bertolotto ${ }^{1}$ \\ ${ }^{1}$ Facultad de Ciencias Exactas y Naturales, Universidad Nacional de La Pampa, Santa Rosa, La Pampa 6300, \\ Argentina \\ ${ }^{2}$ Facultad de Ciencias Exactas, Universidad Nacional de La Plata, La Plata, Buenos Aires B1900BTE, \\ Argentina \\ ${ }^{3}$ Consejo Nacional de Investigaciones Científicas y Técnicas (CONICET), Buenos Aires C1033AAJ, Argentina
}

(Received 10 December 2010; accepted 1 March 2011; published online 30 March 2011)

\begin{abstract}
In the present work, we study the effect of translational-rotational hydrodynamic coupling on the stationary electric linear dichroism of DNA fragments. The theoretical resolution of the problem has, so far, been dealt with analytic methods valid only in the limit of low electric fields. In this work, we apply numerical methods that allow us to study the problem and also consider electric fields of arbitrary strength. We use the bent rod molecules model to describe DNA fragments with physical properties characterized by their electric charge, electric polarizability tensor, rotational diffusion tensor, and translation-rotation coupling diffusion tensor. The necessary orientational distribution function to calculate electric dichroism is obtained by solving the Fokker-Planck equation through the finite difference method. We analyze the different contributions due to electric polarizability and translational-rotational coupling to the electric dichroism. (C) 2011 American Institute of Physics. [doi:10.1063/1.3568270]
\end{abstract}

\section{INTRODUCTION}

Physical properties of macromolecules in solution can be studied through electro-optical techniques. In these procedures, an external electric field is applied on the solution to orient the molecules and induce on it an optical anisotropy. In this way, electric dichroism is induced on the solution, which also manifests itself birefringent. For macromolecules that can be described by simple geometrical shapes, such as cylinders and ellipsoids, the study of the orientation process is relatively simple. In these cases, the molecules orientate themselves due to the interaction between their electric properties (permanent and/or induced electric dipolar moment) with the applied electric field. Once the stationary state is achieved, the orientations can be described with Boltzmann distribution function. However, for asymmetrical shaped particles the analysis is more complex due to the hydrodynamic coupling between their translational and rotational movements. Such coupling for rigid arbitrary shaped particles immersed in a fluid at low Reynolds number has been described by Brenner ${ }^{1}$ some years ago. Nevertheless, the inclusion of hydrodynamic coupling in electro-optical theories is relatively recent. As the torque generated by hydrodynamic coupling is dissipative, the orientational distributional function is not Boltzmannian and must be calculated through the Fokker-Planck equation.

Some advances toward the study of electro-optical effects (electric birefringence and dichroism) of arbitrarily shaped rigid particles have been made. In this sense, Wegener et al. ${ }^{2}$ developed a general formalism for electro-optical effects decay without considering the translation-rotation coupling of molecules. Later, Wegener ${ }^{3,4}$ studied the transient electric

\footnotetext{
a)Electronic mail: pumazano@exactas.unlpam.edu.ar.
}

birefringence, rise and decay of this, including the torque due to the hydrodynamic coupling and derived explicit birefringence expressions for the Kerr limit of static and time dependent weak electric fields. Recently, Kalmykov ${ }^{5}$ developed an alternative approach to the one made by Wegener using a matrix method. Although this method could be, in principle, generalized to incorporate the translation-rotation coupling, this generalization has not been included in his work.

Stationary electric birefringence and dichroism for bent rod DNA fragments have been theoretically studied by Bertolotto and co-workers ${ }^{6-10}$ in the limit of low electric field strengths. They have found that translational-rotational hydrodynamic coupling produces a positive contribution in the electro-optical effects. The requirement for low electric field strengths is originated in the use of approximate analytic methods to solve the Fokker-Planck equation. The effect of the hydrodynamic coupling on electro-optical transients of bent rod DNA fragments was studied through Brownian dynamic simulation by Porschke and co-workers. ${ }^{11-13}$ The existence of a positive contribution on the electro-optical effects due to hydrodynamic coupling is confirmed by its computational simulations. The inclusion of hydrodynamic coupling is decisive to explain why the electric birefringence and dichroism of DNA fragments of relatively long length change its habitual negative sign at low electric field.

In this work, we study the coupling influence between translational and rotational diffusion on the stationary electric dichroism of bent rod DNA fragments orientated by external electric field with arbitrary strength. The bent rods are considered charged and polarizable particles. The orientational distribution function needed to calculate electric dichroism is obtained numerically solving the Fokker-Planck equation through the finite difference method. The individual 
contributions to the reduced electric dichroism of different orientation mechanisms are calculated. Besides, to estimate the error of the numerical calculations, particular cases are analyzed, for which the orientational distribution function is exactly known.

\section{THEORY}

\section{A. Molecular model}

DNA fragments are modeled as rigid bent rod molecules (BRM) geometrically characterized by their arc length $S_{0}$, curvature radius $R$, and bending angle $2 \gamma_{0}$. The BRM is put on the plane $X^{\prime} Z^{\prime}$ of the body coordinate system $O^{\prime} X^{\prime} Y^{\prime} Z^{\prime}$ whose origin $O^{\prime}$ coincides with the BRM center of mass. The molecule electric properties are its electric charge $q$ and its electric polarizability tensor $\left[\alpha^{E}\right]$ which principal elements are

$$
\begin{gathered}
\alpha_{x^{\prime} x^{\prime}}^{E}=2 K R^{2}\left[1-\cos \left(\frac{S_{0}}{R}\right)^{2}\right]^{2} \\
+\frac{1}{2} K a R\left[\sin \left(\frac{S_{0}}{R}\right)+\frac{S_{0}}{R}\right] \\
\alpha_{y^{\prime} y^{\prime}}^{E}=K S_{0} a \\
\alpha_{z^{\prime} z^{\prime}}^{E}=4 K R^{2} \sin ^{2}\left(\frac{S_{0}}{2 R}\right)+\frac{1}{2} K a R\left[\frac{S_{0}}{R}-\sin \left(\frac{S_{0}}{R}\right)\right]
\end{gathered}
$$

where $K$ is an electric polarizability constant and $a$ is the length through which the bounded counterions can be displaced in radial direction in any point of the BRM. This model for electric polarizability of BRM was developed by Bertolotto et al. ${ }^{10}$ The hydrodynamic properties of BRM are given by its rotational diffusion tensor $[R]$ with principal elements $R_{x^{\prime} x^{\prime}}, R_{y^{\prime} y^{\prime}}$, and $R_{z^{\prime} z^{\prime}}$, and the translation-rotation coupling diffusion tensor $[P]$ with nonzero elements $P_{y^{\prime} z^{\prime}}$ and $P_{z^{\prime} y^{\prime}}$. The optical properties are represented by the transition moment of each BRM chromophore group.

\section{B. Orientational distribution function}

The position and orientation of BRM with respect to the laboratory coordinate system $O X Y Z$ are given by the coordinates $x, y, z$ of the $O^{\prime}$ origin of the body coordinate system and the three Euler angles $\phi, \theta$, and $\psi$. We need to obtain the orientational distribution function of particles $f(\boldsymbol{\Omega}, t)$ when the electric field is applied on the solution, where $\boldsymbol{\Omega}$ $=\langle x, y, z, \phi, \theta, \psi\rangle$. The $Z$ axis direction is arbitrarily chosen matching the direction of the applied electric field. The distribution function $f(\boldsymbol{\Omega}, t)$ is determined by the Fokker-Planck equation of the system

$$
\frac{\partial f(\boldsymbol{\Omega}, t)}{\partial t}=-\frac{1}{\sqrt{g}} \vec{\nabla}_{\boldsymbol{\Omega}}[\sqrt{g} \mathbf{J}(\boldsymbol{\Omega}, t)],
$$

where $\quad \vec{\nabla}_{\boldsymbol{\Omega}}=\langle\partial / \partial x, \partial / \partial y, \partial / \partial z, \partial / \partial \phi, \partial / \partial \theta, \partial / \partial \psi\rangle, \quad g$ $=\sin ^{2} \theta$ is the determinant of the metric tensor, and $\mathbf{J}(\boldsymbol{\Omega}, t)$ is the probability current which depends on the derive and diffusion moments $\mathbf{M}_{1}$ and $\left[M_{2}\right]$, respectively, in the following way:

$$
\mathbf{J}(\boldsymbol{\Omega}, t)=\mathbf{M}_{1} f(\boldsymbol{\Omega}, t)-\frac{1}{2} \vec{\nabla}_{\boldsymbol{\Omega}}\left(\left[M_{2}\right] f(\boldsymbol{\Omega}, t)\right) .
$$

In the following lines, we will make some assumptions to simplify the number of variables in our problem. We suppose that, in the time in which the rotational equilibrium is reached, the electroforetic migration does not modify the molecules spatial concentration. Thus, the orientational distribution function is independent from the position coordinates. Besides, as the electric field introduces a cylindrical symmetry in the system, $f$ also results independent from $\phi$ angle. Under these simplifications, Eq. (4) can be developed introducing a local coordinate system and then transforming it into a laboratory coordinate system. Details about this calculation were described by Bertolotto 9 and will be omitted in this work. Through the above-mentioned procedure the Fokker-Planck equation in the stationary state results

$$
\begin{gathered}
\left(Q_{\theta \theta} \frac{\partial^{2}}{\partial \theta^{2}}+Q_{\psi \psi} \frac{\partial^{2}}{\partial \psi^{2}}+Q_{\psi \theta} \frac{\partial^{2}}{\partial \psi \partial \theta}+Q_{\theta} \frac{\partial}{\partial \theta}\right. \\
\left.+Q_{\psi} \frac{\partial}{\partial \psi}+Q_{00}\right) f(\theta, \psi)=0,
\end{gathered}
$$

where

$$
\begin{gathered}
Q_{\theta \theta}=\left(R_{x^{\prime} x^{\prime}} \cos ^{2} \psi+R_{y^{\prime} y^{\prime}} \sin ^{2} \psi\right), \\
Q_{\psi \psi}=\left(R_{x^{\prime} x^{\prime}} \sin ^{2} \psi \cot ^{2} \theta+R_{y^{\prime} y^{\prime}} \cos ^{2} \psi \cot ^{2} \theta+R_{z^{\prime} z^{\prime}}\right), \\
Q_{\psi \theta}=2\left(R_{y^{\prime} y^{\prime}}-R_{x^{\prime} x^{\prime}}\right) \sin \psi \cos \psi \cot \theta, \\
Q_{\theta}=\cot \theta\left(R_{x^{\prime} x^{\prime}} \sin ^{2} \psi+R_{y^{\prime} y^{\prime}} \cos ^{2} \psi\right) \\
-\frac{E^{2}}{k_{B} T} \cos \theta \sin \theta\left[\left(\alpha_{y^{\prime} y^{\prime}}^{E}-\alpha_{z^{\prime} z^{\prime}}^{E}\right) R_{x^{\prime} x^{\prime}} \cos ^{2} \psi,\right. \\
\left.-\left(\alpha_{z^{\prime} z^{\prime}}^{E}-\alpha_{x^{\prime} x^{\prime}}^{E}\right) R_{y^{\prime} y^{\prime}} \sin ^{2} \psi\right]+\frac{q E}{k_{B} T} P_{y^{\prime} z^{\prime}} \cos \theta \sin \psi,
\end{gathered}
$$

$$
\begin{aligned}
Q_{\psi}= & \frac{\cos \psi}{\sin ^{2} \theta}\left\{\frac { E ^ { 2 } } { k _ { B } T } \left[R_{z^{\prime} z^{\prime}}\left(\alpha_{y^{\prime} y^{\prime}}^{E}-\alpha_{x^{\prime} x^{\prime}}^{E}\right) \sin ^{2} \theta\right.\right. \\
& \left.+R_{y^{\prime} y^{\prime}}\left(\alpha_{z^{\prime} z^{\prime}}^{E}-a_{x^{\prime} x^{\prime}}^{E}\right) \cos ^{2} \theta+R_{x^{\prime} x^{\prime}}\left(\alpha_{y^{\prime} y^{\prime}}^{E}-\alpha_{z^{\prime} z^{\prime}}^{E}\right) \cos ^{2} \theta\right] \\
& \times \sin ^{2} \theta \sin \psi+\frac{E q}{k_{B} T} \sin \theta\left(P_{y^{\prime} z^{\prime}} \cos ^{2} \theta-P_{z^{\prime} y^{\prime}} \sin ^{2} \theta\right) \\
& \left.+\left(R_{x^{\prime} x^{\prime}}-R_{y^{\prime} y^{\prime}}\right) \sin \psi\left(\cos ^{2} \theta+1\right)\right\},
\end{aligned}
$$

$$
\begin{aligned}
Q_{00}= & -\frac{1}{\sin \theta}\left\{\frac{q E}{k_{B} T}\left(P_{y^{\prime} z^{\prime}}-P_{z^{\prime} y^{\prime}}\right) \sin \psi \sin ^{2} \theta\right. \\
& +\frac{E^{2}}{k_{B} T} R_{z^{\prime} z^{\prime}}\left(\alpha_{x^{\prime} x^{\prime}}^{E}-\alpha_{y^{\prime} y^{\prime}}^{E}\right) \sin ^{3} \theta\left(2 \cos ^{2} \psi-1\right) \\
& +\frac{E^{2}}{k_{B} T} \sin \theta\left[R_{x^{\prime} x^{\prime}}\left(\alpha_{y^{\prime} y^{\prime}}^{E}-\alpha_{z^{\prime} z^{\prime}}^{E}\right)\left(\cos ^{2} \theta-\sin ^{2} \theta \cos ^{2} \psi\right)\right. \\
& \left.\left.\times R_{y^{\prime} y^{\prime}}\left(\alpha_{z^{\prime} z^{\prime}}^{E}-\alpha_{x^{\prime} x^{\prime}}^{E}\right)+\left(\sin ^{2} \theta \sin ^{2} \psi-\cos ^{2} \theta\right)\right]\right\} .
\end{aligned}
$$


In order to solve Eq. (6), the function $f$ will be expressed in the following way:

$$
f(\theta, \psi)=f^{0}+f^{E}(\theta, \psi),
$$

where $f^{0}$ is the orientational distribution function in absence of applied electric field and $f^{E}(\theta, \psi)$ represents the modifications due to the presence of electric field. Replacing Eq. (7) in Eq. (6), we obtain

$$
\begin{array}{r}
\left(Q_{\theta \theta} \frac{\partial^{2}}{\partial \theta^{2}}+Q_{\psi \psi} \frac{\partial^{2}}{\partial \psi^{2}}+Q_{\psi \theta} \frac{\partial^{2}}{\partial \psi \partial \theta}+Q_{\theta} \frac{\partial}{\partial \theta}\right. \\
\left.+Q_{\psi} \frac{\partial}{\partial \psi}+Q_{00}\right) f^{E}=-Q_{00} f^{0} .
\end{array}
$$

Here $f^{0}$ is simply a constant because when the electric field is not present all orientations are equally probable. Therefore, the orientational distribution function is determined by solving the differential equation given by Eq. (8) for $f^{E}(\theta, \psi)$. To do this, we use the finite difference method (FDM). ${ }^{14}$ First, we make a grid of discrete points $\theta_{l}, \psi_{m}$ on the domain $0 \leq \theta \leq \pi$ and $0 \leq \psi \leq 2 \pi$. The number of points in the $\theta$ and $\psi$ directions are denominated by $n_{\theta}$ and $n_{\psi}$, respectively. Each interior grid point is generated by the following relations:

$$
\begin{array}{ll}
\theta_{l}=\theta_{l-1}+\Delta \theta, & l=1,2, \ldots, n_{\theta}-2 \\
\psi_{m}=\psi_{m-1}+\Delta \psi, & m=1,2, \ldots n_{\psi}-2
\end{array}
$$

with $\theta_{0}=0, \psi_{0}=0, \theta_{n_{\theta}-1}=\pi$, and $\psi_{n_{\psi}-1}=2 \pi$. The mesh size in the $\theta$ and $\psi$ directions are $\Delta \theta=\pi /\left(n_{\theta}-1\right)$ and $\Delta \psi=2 \pi /\left(n_{\psi}-1\right)$, respectively. Second, using central differences, we obtain the following approximations for the values that are assumed by $f^{E}$ in the grid point

$$
\begin{gathered}
\left.\frac{\partial f^{E}}{\partial \theta}\right|_{l, m} \approx \frac{f_{l+1, m}^{E}-f_{l-1, m}}{2 \Delta \theta}, \\
\left.\frac{\partial f^{E}}{\partial \psi}\right|_{l, m} \approx \frac{f_{l, m+1}^{E}-f_{l, m-1}^{E}}{2 \Delta \psi}, \\
\left.\frac{\partial^{2} f^{E}}{\partial \theta^{2}}\right|_{l, m} \approx \frac{f_{l+1, m}^{E}-2 f_{l, m}^{E}+f_{l-1, m}^{E}}{\Delta \theta^{2}}, \\
\left.\frac{\partial^{2} f^{E}}{\partial \psi^{2}}\right|_{l, m} \approx \frac{f_{l, m+1}^{E}-2 f_{l, m}^{E}+f_{l, m-1}^{E}}{\Delta \psi^{2}},
\end{gathered}
$$

$$
\begin{aligned}
\left.\frac{\partial^{2} f^{E}}{\partial \psi \partial \theta}\right|_{l, m} \approx & \frac{1}{2 \Delta \theta \Delta \psi}\left(f_{l+1, m+1}^{E}+f_{l-1, m-1}^{E}+2 f_{l, m}^{E}\right. \\
& \left.-f_{l+1, m}^{E}-f_{l-1, m}^{E}-f_{l, m+1}^{E}-f_{l, m-1}^{E}\right),
\end{aligned}
$$

where subscripts $i, j$, with $i=l, l \pm 1$ and $j=m, m \pm 1$, that accompany $f^{E}$ mean that the function must be evaluated at the point $\theta_{i}, \psi_{j}$. We will keep this nomenclature for the rest of the work.

Using these approximations for the derivates, Eq. (8) results in the following relation:

$$
\begin{aligned}
H_{1} f_{l+1, m}^{E} & +H_{2} f_{l, m}^{E}+H_{3} f_{l-1, m}^{E}+H_{4} f_{l, m+1}^{E} \\
& +H_{5} f_{l, m-1}^{E}+H_{6} f_{l+1, m+1}^{E}+H_{7} f_{l-1, m-1}^{E}=H_{8} f^{0},
\end{aligned}
$$

where the coefficients $H_{i}$ are given by

$$
\begin{gathered}
H_{1}=\frac{Q_{\theta \theta}}{\Delta \theta^{2}}-\frac{Q_{\psi \theta}}{2 \Delta \theta \Delta \psi}+\left.\frac{Q_{\theta}}{2 \Delta \theta}\right|_{l, m}, \\
H_{2}=-\frac{2 Q_{\theta \theta}}{\Delta \theta^{2}}-\frac{2 Q_{\psi \psi}}{\Delta \psi^{2}}+\frac{Q_{\psi \theta}}{\Delta \theta \Delta \psi}+\left.Q_{00}\right|_{l, m}, \\
H_{3}=\frac{Q_{\theta \theta}}{\Delta \theta^{2}}-\frac{Q_{\psi \theta}}{2 \Delta \theta \Delta \psi}-\left.\frac{Q_{\theta}}{2 \Delta \theta}\right|_{l, m}, \\
H_{4}=\frac{Q_{\psi \psi}}{\Delta \psi^{2}}-\frac{Q_{\psi \theta}}{2 \Delta \theta \Delta \psi}+\left.\frac{Q_{\psi}}{2 \Delta \psi}\right|_{l, m}, \\
H_{5}=\frac{Q_{\psi \psi}}{\Delta \psi^{2}}-\frac{Q_{\psi \theta}}{2 \Delta \theta \Delta \psi}-\left.\frac{Q_{\psi}}{2 \Delta \psi}\right|_{l, m}, \\
H_{6}=\left.\frac{Q_{\psi \theta}}{2 \Delta \theta \Delta \psi}\right|_{l, m} \\
H_{7}=H_{6}, \\
H_{8}=-\left.Q_{00}\right|_{l, m} .
\end{gathered}
$$

When we vary subscript $l$ from one to $n_{\theta}-2$ and $m$ form one to $n_{\psi}-2$, Eq. (9) generates a system of $\left(n_{\theta}-2\right) \times\left(n_{\psi}-2\right)$ linear equations for the $n_{\theta} \times n_{\psi}$ values that adopt function $f^{E}$ in the grid points. The remaining equations necessary to have a system with the same amount of equations and unknowns are given by the boundary conditions. Such conditions are established in terms of the probability current vector whose components in the $\theta$ and $\psi$ directions are given by

$$
\begin{gathered}
J_{\theta}(\theta, \psi)=\frac{E}{k_{B} T} \sin \theta \cos \theta f\left\{\left(\alpha_{y^{\prime} y^{\prime}}^{E}-\alpha_{z^{\prime} z^{\prime}}^{E}\right) E R_{x^{\prime} x^{\prime}} \cos ^{2} \psi \sin \theta+\sin \psi\left[-P_{y^{\prime} z^{\prime}} q+\left(\alpha_{x^{\prime} x^{\prime}}^{E}-\alpha_{z^{\prime} z^{\prime}}\right) E R_{y^{\prime} y^{\prime}} \sin \theta \sin \psi\right]\right\} \\
+\sin \theta\left[\left(R_{x^{\prime} x^{\prime}}-R_{y^{\prime} y^{\prime}}\right) \cos \psi \cot \theta \sin \psi \frac{\partial f}{\partial \psi}-\left(R_{x^{\prime} x^{\prime}} \cos ^{2} \psi+R_{y^{\prime} y^{\prime}} \sin ^{2} \psi\right) \frac{\partial f}{\partial \theta}\right] \\
J_{\psi}(\theta, \psi)=\frac{E}{k_{B} T} \sin \theta \cos \psi f\left\{-P_{y^{\prime} z^{\prime}} q \cos \theta \cot \theta-E\left[\left(\alpha_{y^{\prime} y^{\prime}}^{E}-\alpha_{z^{\prime} z^{\prime}}\right) R_{x^{\prime} x^{\prime}}+\left(\alpha_{z^{\prime} z^{\prime}}^{E}-\alpha_{x^{\prime} x^{\prime}}\right) R_{y^{\prime} y^{\prime}}\right]\right. \\
\\
\left.\cos ^{2} \theta \sin \psi+\sin \theta\left[P_{z^{\prime} y^{\prime}} q+\left(\alpha_{x^{\prime} x^{\prime}}^{E}-\alpha_{y^{\prime} y^{\prime}}^{E}\right) E R_{z^{\prime} z^{\prime}} \sin \theta \sin \psi\right]\right\}-\sin \theta \\
\left(R_{z^{\prime} z^{\prime}}+R_{y^{\prime} y^{\prime}} \cos ^{2} \psi \cot ^{2} \theta+R_{x^{\prime} x^{\prime}} \cot ^{2} \theta \sin ^{2} \psi\right) \frac{\partial f}{\partial \psi}+\left(R_{x^{\prime} x^{\prime}}-R_{y^{\prime} y^{\prime}}\right) \cos \theta \cos \psi \sin \psi \frac{\partial f}{\partial \theta} .
\end{gathered}
$$


The boundary conditions for $\theta$ are $J_{\theta}(\theta=0, \psi)=0$ and $J_{\theta}(\theta=\pi, \psi)=0$. By using Eq. (7) and applying the central difference approximation these conditions are given by

$$
\begin{aligned}
& f_{0, m+1}^{E}-f_{0, m-1}^{E}=0, m=0,1,2, \ldots, n_{\psi}-1, \\
& f_{n_{\theta}-1, m+1}^{E}-f_{n_{\theta}-1, m-1}^{E}=0, m=0,1,2, \ldots, n_{\psi}-1 .
\end{aligned}
$$

Let us note that in the last two equations we vary $m$ from 0 to $n_{\psi}-1$. This means that we have introduced dummy grid points, in the sense that they do not belong to the domain of the problem. The dummy points correspond to grid expansions toward points with coordinates $\psi=\psi_{0}-\Delta \psi$ and $\psi=\psi_{n_{\psi}-1}+\Delta \psi$, where the function has values $f_{l,-1}^{E}$ and $f_{l, n_{\psi}}^{E}$. These dummy values of $f^{E}$ add $2 n_{\psi}$ unknowns to our problem that will be eliminated after we solve the equation system because they lack physical meaning.

For the $\psi$ variable, we use periodic boundary conditions because the moments $\mathbf{M}_{1}$ and $\left[M_{2}\right]$ are periodic functions in $\psi$ with period $2 \pi .{ }^{15}$ This implies that $f(\theta, \psi=0)$ $=f(\theta, \psi=2 \pi)$ and $J_{\psi}(\theta, \psi=0)=J_{\psi}(\theta, \psi=2 \pi)$. Applying the central difference approximation and using Eq. (7) the mentioned conditions take us to the following two relations:

$$
\begin{aligned}
& f_{l, 0}^{E}-f_{l, n_{\psi}-1}^{E}=0, \quad l=0,1,2, \ldots, n_{\theta}-1, \\
& \frac{1}{4 \Delta \psi}\left[R_{y^{\prime} y^{\prime}}+R_{z^{\prime} z^{\prime}}+\left(R_{y^{\prime} y^{\prime}}-R_{z^{\prime} z^{\prime}}\right) \cos (2 \theta)\right] \\
& \quad \times\left(f_{l,-1}^{E}-f_{l, 1}^{E}-f_{l, n_{\psi}-2}+f_{l, n_{\psi}}^{E}\right) \\
& \quad+\frac{q E}{k_{B} T}\left[P_{z^{\prime} y^{\prime}} \sin ^{2} \theta-P_{y^{\prime} z^{\prime}} \cos ^{2} \theta\right] \sin \theta \\
& \quad \times\left(f_{l, 0}^{E}-f_{l, n_{\psi}-1}^{E}\right)=0 \quad l=0,1, \ldots, n_{\theta}-1 .
\end{aligned}
$$

Let us note that when we expand the grid and add the dummy points, Eq. (9) generates now $\left(n_{\theta}-2\right) \times n_{\psi}$ equations that are generated doing $l=1,2, \ldots, n_{\theta}-2$ and $m=0,1,2, \ldots, n_{\psi}-1$. Our problem is reduced, then, to solve the system of $n_{\theta} \times\left(n_{\psi}+2\right)$ equations generated by Eqs. (9)-(13) to know the $n_{\theta} \times\left(n_{\psi}+2\right)$ values of $f_{l, m}^{E}$ that the function adopts in the grid points, included the dummy ones.

Finally, to have a probability being equal to 1 for finding a particle with any orientation inside a solid angle $8 \pi^{2}$, the orientational distribution function that we obtain must be normalized in the following way:

$$
f(\theta, \psi)=\frac{f^{0}+f^{E}(\theta, \psi)}{2 \pi \int_{0}^{2 \pi} \int_{0}^{\pi}\left(f^{0}+f^{E}\right) \sin \theta d \theta d \psi}
$$

As $f^{0}$ is an arbitrary constant, we choose $f^{0}=1$ to make the calculations.

\section{Reduced electric linear dichroism}

The electric reduced dichroism (RELD) is defined as

$$
\frac{\Delta A}{A}=\frac{A_{/ /}-A_{\perp}}{A},
$$

where $A_{/ /}$and $A_{\perp}$ are the solution absorbances for linearly polarized incident light according to the parallel and perpendicular directions of the applied electric field, and $A$ is the absorbance of the solution for randomly oriented molecules. The subscripts // and $\perp$ will be kept for the rest of the work with the same meaning used above.

In the present work, we will deduce an expression for RELD of bent rod DNA fragments carrying out a similar treatment to the one applied by Bertolotto et al. ${ }^{10}$ The procedure is based on a work by Schellman. ${ }^{16}$ The author used quantum theory of absorption to determine that the extinction coefficient and, therefore, the absorbance of a system of monomer units with fixed orientation is proportional to $|\mathbf{p} \cdot \mathbf{E}|^{2}$, where $\mathbf{p}$ is the monomer unit transition moment and $\mathbf{E}$ is the electric field of the incident light. This result can be applied to system of polymers without fixed orientation. Supposing that the interaction among the different chromophore groups (monomer units) in the polymer is weak enough as to omit the interaction between them, the reduced electric dichroism results

$$
\frac{\Delta A}{A}=\frac{\left\langle w_{/ /}\right\rangle-\left\langle w_{\perp}\right\rangle}{\langle w\rangle},
$$

where

$$
\begin{gathered}
w_{/ /}=\sum_{i=1}^{N}\left|\mathbf{p}_{i} \cdot \mathbf{E}_{/ /}\right|^{2}, \\
w_{\perp}=\sum_{i=1}^{N}\left|\mathbf{p}_{i} \cdot \mathbf{E}_{\perp}\right|^{2}, \\
w=\sum_{i=1}^{N}\left|\mathbf{p}_{i} \cdot \mathbf{E}\right|^{2} .
\end{gathered}
$$

The summatories in the above equations are over all the chromophore groups in the BRM. The brackets in Eq. (16) mean statistical average on all the orientations. $w_{/ /}$and $w_{\perp}$ must be averaged having into account that there is an applied electric field while $w$ must be averaged for random orientation. In the last case, it is the same to consider linearly polarized light according to the parallel or perpendicular directions to the applied electric field, therefore no subscript // or $\perp$ has been included in Eq. (19).

Being $X^{\prime \prime} Y^{\prime \prime} Z^{\prime \prime}$ a system of coordinates with its $Z^{\prime \prime}$ axis tangent at the arc in the point at which the chromophore group $i$ is located (Fig. 1). Transition moment $\mathbf{p}_{i}$, in this system, is given by

$$
\mathbf{p}_{i}=(p \cos \beta, p \sin \beta, 0),
$$

where $\beta$ is the angle that projects $\mathbf{p}_{i}$ on the axes $X^{\prime \prime}$ and $Y^{\prime \prime}$, respectively. The component of $\mathbf{p}_{i}$ according to $Z^{\prime \prime}$ is 0 because the electronic transitions are produced in the DNA basis 


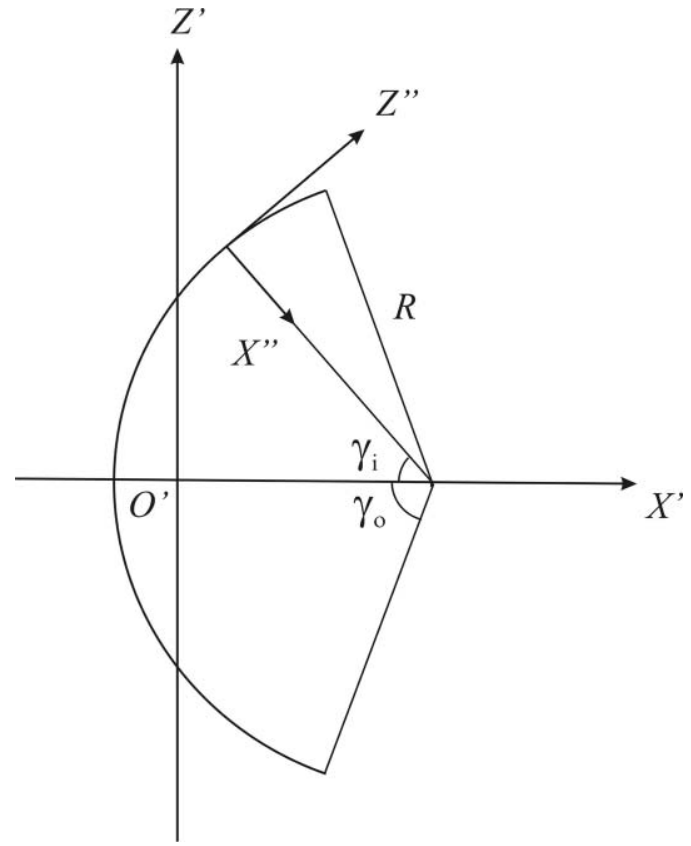

FIG. 1. Bent rod molecule in the body coordinate system.

plane. In the body coordinate system, the transition moment of the chromophore group $i$ is

$$
\mathbf{p}_{i}=\left(p \cos \beta \sin \gamma_{i}, p \sin \beta,-p \cos \beta \sin \gamma_{i}\right) .
$$

We transform Eq. (21) to the laboratory coordinate system with the Euler transformation matrix and develop Eqs. (17)-(19) already mentioned in the coordinate system. To do this, the angle $\gamma_{i}$ is expressed as $\gamma_{i}=-\gamma_{0}+(i$ $-1 / 2) b / R$, where $b$ is the distance between two consecutive chromophore groups in the BRM. The result is averaged in the angle $\beta$ with probability density $1 / 2 \pi$ to consider that the transition moments are randomly oriented in the basis plane. Then, we applied the approximation $\cot (b / R) \cong R / b$ in the obtained expressions for $w_{/ /}, w_{\perp}$, and $w$, and replaced them in Eq. (16). Thus, the RELD results

$$
\begin{aligned}
\frac{\Delta A}{A}= & \frac{3 \pi}{16 \gamma_{o}} \int_{0}^{2 \pi} \int_{0}^{\pi}\left[\left(-\gamma_{o}-3 A_{o}\right)(1+3 \cos 2 \theta)\right. \\
& \left.-6\left(-\gamma_{o}+A_{o}\right) \cos 2 \psi \sin ^{2} \theta\right] f(\theta, \psi) \sin \theta d \theta d \psi,
\end{aligned}
$$

where $A_{o}=\cos \gamma_{o} \sin \gamma_{o}$.

In Eq. (22) the statistical average in the angle $\phi$ has been evaluated. This can be done because the orientational distribution function is independent from this angle.

\section{RESULTS AND DISCUSSION}

First, we calculated the RELD of DNA fragments of $179 \mathrm{pb}$ using the BRM model with a bending angle of $116^{\circ}$, an arc length of $608.6 \AA$ and a diameter of $24 \AA$. The hydrodynamic parameters used to characterize the fragments were obtained applying the Hydro++ program by Garcia de la Torre. ${ }^{17}$ To know the orientational distribution function we
TABLE I. Principal elements of $\left[\alpha^{E}\right]$ tensor.

\begin{tabular}{lccc}
\hline $2 \gamma_{0}(\mathrm{deg})$ & $\alpha_{x^{\prime} x^{\prime}}^{E} \times 10^{17}\left(\mathrm{~cm}^{3}\right)$ & $\alpha_{y^{\prime} y^{\prime}}^{E} \times 10^{17}\left(\mathrm{~cm}^{3}\right)$ & $\alpha_{z^{\prime} z^{\prime}}^{E} \times 10^{16}\left(\mathrm{~cm}^{3}\right)$ \\
\hline 120 & 8.523 & 6.086 & 2.712 \\
116 & 8.387 & 6.086 & 2.769 \\
100 & 7.863 & 6.086 & 2.987 \\
80 & 7.269 & 6.086 & 3.229 \\
60 & 6.772 & 6.086 & 3.430 \\
\hline
\end{tabular}

solved the Fokker-Planck equation through the FDM as was described in Sec. II B using a grid with $n_{\theta}=n_{\psi}=75$. The electric polarizability constant used is $K=1 \times 10^{-5} \mathrm{~cm}$ and the value assigned to the electric charge is $q=0.16 Q$, where $Q$ is the DNA fragment charge when all the phosphate groups are ionized. The electric polarizability values corresponding to that assigned to $K$ are shown in Table I for the different bending angles that are used in the present work.

Figure 2 shows stationary RELD values as function of the applied electric field for BRM with the above mentioned parameters. Figure 2 also shows the individual contributions to RELD for orientations only due to the induced electric dipolar moment-electric field interaction and only to the translation-rotation hydrodynamic coupling. In the first case, the molecules tend to orientate in parallel direction to the electric field which gives us a negative dichroism component. In the second case, the molecules tend to orientate in perpendicular direction to the electric field and thus, the dichroism is positive due to hydrodynamic coupling. This positive component causes the inversion of the habitual negative sign in the electric dichroism at low electric field. When the electric field strength rises, the electric dichroism reaches negative values associated to an intense component due to electric polarizability. However, we observe that the contribution due to hydrodynamic coupling remains in all the electric field strength range that is shown in Fig. 2. The results clearly show that the contributions to RELD originated in the orientation due to electric polarizability and hydrodynamic coupling are not additive.

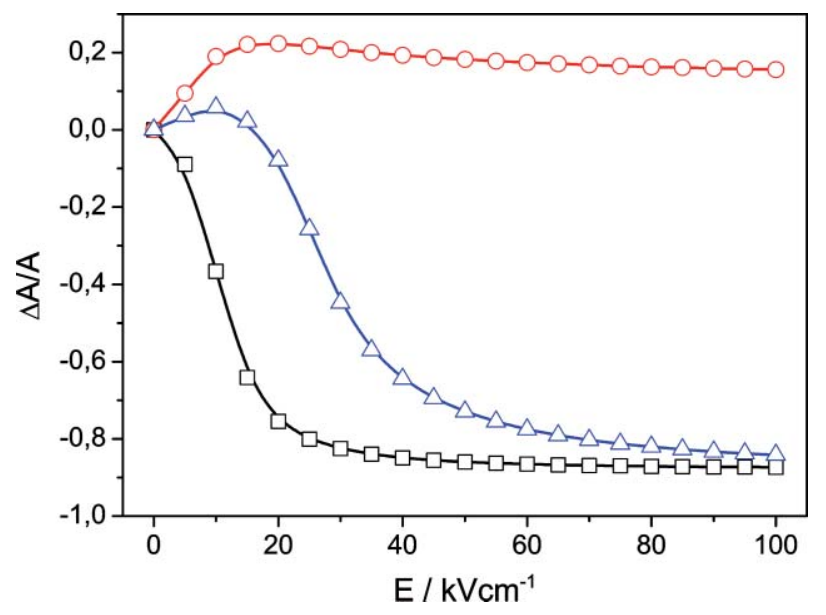

FIG. 2. Stationary RELD as function of the electric field strength of BRM solution. (- $\square$-) Orientation due to electric polarizability, (-०-) orientation due to hydrodynamic coupling, and $\left(-\Delta_{-}\right)$orientation due to electric polarizability and hydrodynamic coupling combined. 


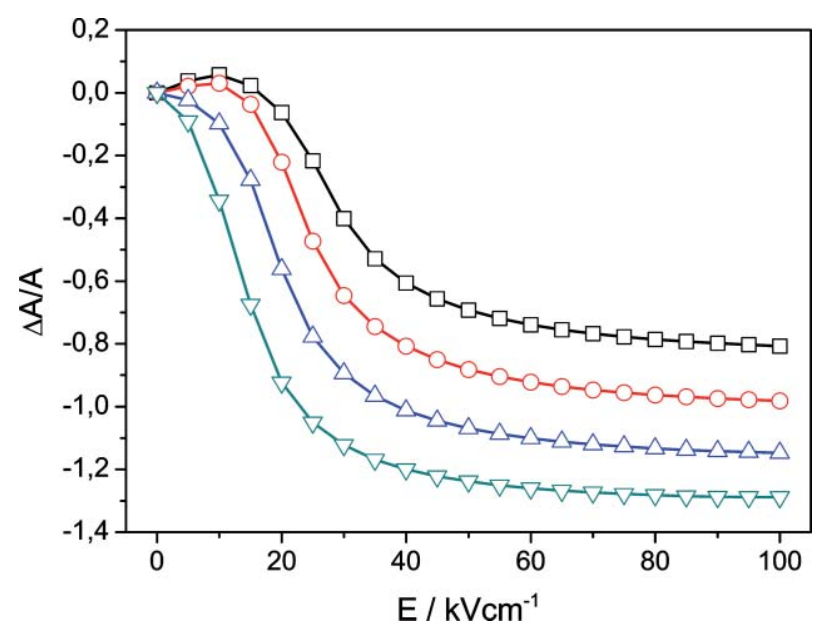

FIG. 3. Stationary RELD as a function of the electric field strength of BRM with different bending angles. (- $\square-) 120^{\circ}$, (-०-) $100^{\circ},\left(-\Delta^{-}\right) 80^{\circ},(-\nabla-) 60^{\circ}$.

In Fig. 3 we observe the effect of changing the BRM bending angle on the electric dichroism. When the bending angle rises, the positive component of the electric dichroism associated to hydrodynamic coupling becomes more important. Consistently, we observe that for higher bending angles $\left(100^{\circ}\right.$ and $\left.120^{\circ}\right)$ the electric dichroism at low electric field is positive while for lower bending angles $\left(60^{\circ}\right.$ and $\left.80^{\circ}\right)$ the electric dichroism is negative in all the range of electric field analyzed. The latter does not mean that it does not exist a contribution due to translation-rotation coupling for bending angles equal to $60^{\circ}$ and $80^{\circ}$ but that the mentioned contribution is not intense enough as to achieve the sign inversion in the electro-optical signal.

In Fig. 4 we show the results obtained for RELD as a function of the electric field for BRM with the same orientational parameters, i.e., the same conformation, electric charge, and electric polarizability, used by Antosiewicz and Porschke $^{12}$ together with the results obtained by them through Brownian dynamic simulation. Figure 4 shows that the results

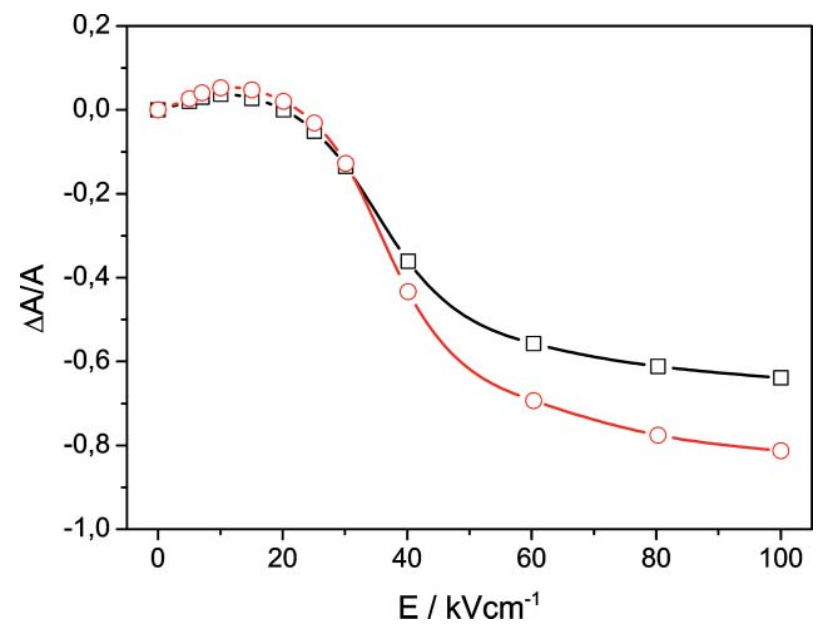

FIG. 4. Stationary RELD as function of the electric field strength of BRM solution whit the same orientational parameters used by Porschke and Antosiewicz. $^{12}$ (- $\square$-) Values obtained by Porschke and Antosiewicz ${ }^{12}$ with Brownian dynamic simulation, (-o-) values obtained by solving FokkerPlanck equation with MDF.

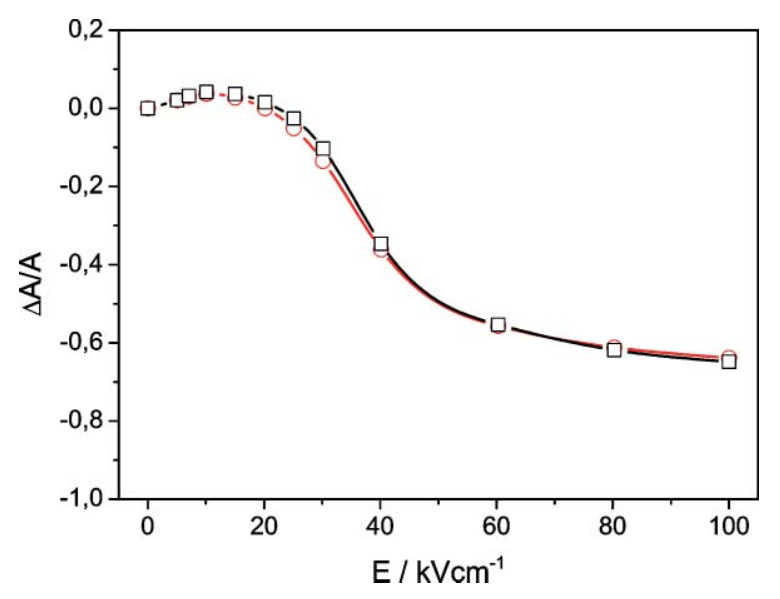

FIG. 5. Stationary RELD as function of the electric field strength of BRM solution whit the same orientational and optical parameters used by Porschke and Antosiewicz ${ }^{12}$. (- $\left.\square-\right)$ Values obtained by Porschke and Antosiewicz ${ }^{12}$ with Brownian dynamic simulation, (-o-) values obtained solving FokkerPlanck equation with MDF.

from both calculations qualitatively agree. However, there is an apparently quantitative disagreement that will be explained in the following lines. Although the orientation process is the same in both calculations, the optical characterization of BRM is not exactly equal. Antosiewicz and Porschke ${ }^{12}$ used an extinction coefficient tensor which elements in the body coordinate system are $\varepsilon_{x^{\prime}}=0.35507, \varepsilon_{y^{\prime}}=0.4665$, and $\varepsilon_{z^{\prime}}=0.17842$ (normalized to trace $=1$ ). These authors estimated these values based on a previous work. ${ }^{18}$ In the present work, we characterized the optical properties of BRM using the transition moment $\mathbf{p}$ of each chromophore group [Eq. (20)]. Equivalently, we can describe the BRM with its extinction coefficient, which is proportional to the sum of $\mathbf{p}^{t} \mathbf{p}$ over all the chromophore groups, where the superscript $t$ means transpose. For our model, developed considering that the BRM is a discrete chain of chromophore units, each of which is described with a transition moment that is randomly oriented in the corresponding basis plane, the elements of extinction coefficient tensor are $\varepsilon_{x^{\prime}}=0.36098, \varepsilon_{y^{\prime}}=0.5$, and $\varepsilon_{z^{\prime}}=0.13901$ (normalized to trace $=1$ ). These values are slightly different than those used by Antosiewicz and Porschke. $^{12}$ If we, besides using the same orientational parameters than Antosiewicz and Porschke, ${ }^{12}$ use the same extinction coefficient tensor, the results of both calculation procedures really agree (Fig. 5). In this way, the study of the orientation process here developed coincides with those of Antosiewicz and Porschke, ${ }^{12}$ being the differences of RELD values attributed to use of different optical parameters to describe the BRM. Nowadays, the question about which optical model is more adequate to describe DNA fragments is difficult to answer. To conclude about this point, it would be necessary to have experimental data of saturation electric dichroism of short DNA fragments in aqueous solution.

The two curves shown in Fig. 5 represent the result to the same problem studied by different methods: theory and computational simulation. Besides differing in the method of study both works differ in the reference system used to describe the physical problem. In the present work, the body 


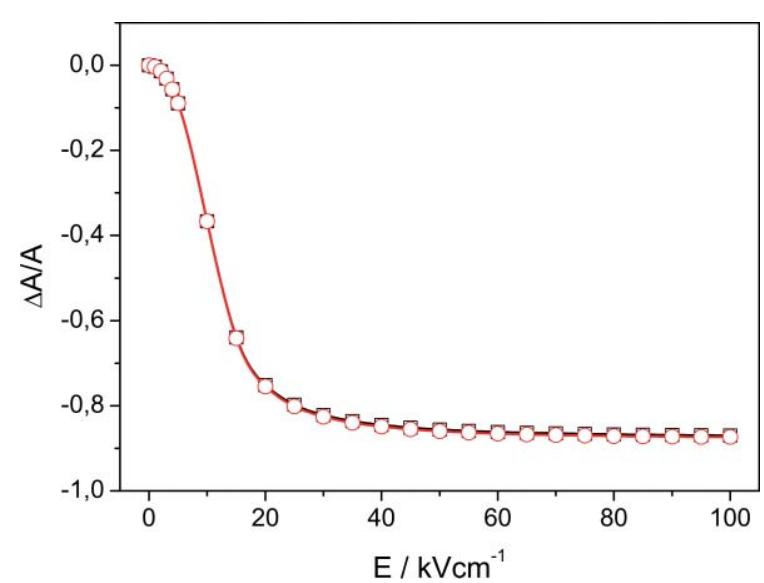

FIG. 6. Stationary RELD as function of the electric field strength of BRM solution with the following parameters: $q=0$ and $K=1 \times 10^{-5} \mathrm{~cm}$. (- $\square-$ ) Values obtained by using the Boltzmann distribution function, (-o-) values obtained solving Fokker-Planck equation for the distribution function.

coordinate system is chosen with its origin in the center of mass while Antosiewicz and Porschke ${ }^{12}$ use the origin in the center of diffusion. It is convenient to make some considerations in respect to the election of the origin of the body coordinate system. For DNA fragments it is habitual to consider that the centers of positive and negative charges agree with the center of mass. This means that the molecules are considered nonpolar and that they lack permanent electric dipolar moment. However, as the system is not neutral, there exists a permanent electric moment, or permanent electric dipolar quasimoment, non-null if we calculate it with respect to a system with a different origin to the center of charge. ${ }^{19}$ In the present work, the origin of the body coordinates system coincides with the center of charge of the BRM and, therefore, no electric moment has been considered. On the contrary, Antosiewicz and Porschke ${ }^{12}$ use the body coordinate system with origin in the center of diffusion and that is why they include this quasipermanent moment. The equivalence between both descriptions was proved by Bertolotto. ${ }^{9} \mathrm{He}$ found that the contribution to RELD of translation-rotation coupling of molecules with the origin of the body coordinate system in their center of charge results, when the origin of the body coordinate system coincides with the center of diffusion, in an electric moment contribution that is balanced by the changing of the translation-rotation coupling tensor due to the change of the origin. The concordance between the results shown in Fig. 5 corroborates the equivalence of both elections for the origin of the body coordinate system.

The numerical calculation procedure for RELD implemented in this work is a practical alternative to determine molecular parameters fitting experimental curves of RELD as a function of electric field strength. For this purpose, the numerical calculation procedure here developed is more convenient that computational simulation because the first one is less time consuming.

To estimate the error in the numeric calculation when we solve the Fokker-Planck equation through FDM, we studied the particular case in which $q=0$. Under this circumstance, the system is conservative and the distribution function in

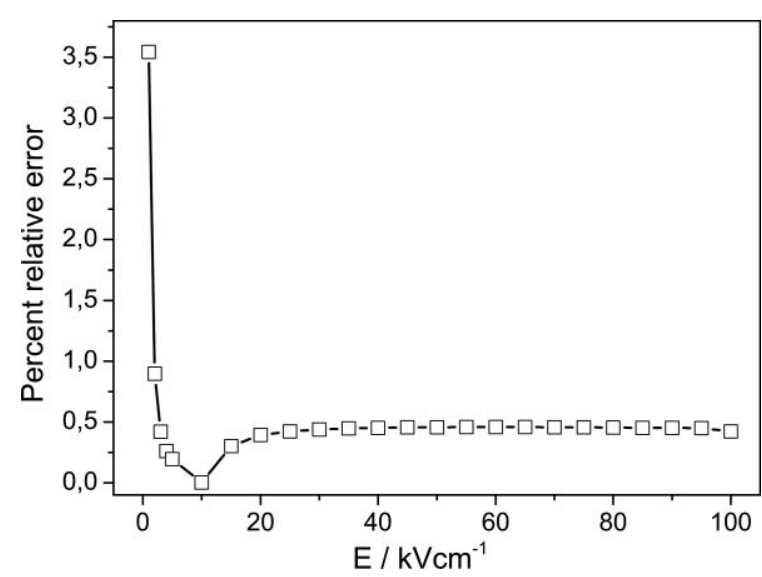

FIG. 7. Absolute value of percent relative error between the results of RELD shown in Fig. 5.

the stationary state is the Boltzmann distribution function. Figure 6 shows that RELD curves calculated employing the Boltzmann distribution function and the numerical solution of the Fokker-Planck equation agree well enough in the electric field strength range studied. The percent relative error is shown in Fig. 7. This is maximum for the lower electric field strength $(1 \mathrm{kV} / \mathrm{cm})$ and rapidly decreases when the applied electric field rises. In this way, the error in the numerical calculation is comparable to the experimental errors.

The numeric calculation of RELD was also proved to reproduce the values obtained through the analytic calculations reported by Bertolotto et al. ${ }^{10}$ for $q \neq 0$ in the limit of low electric fields (up to $2 \mathrm{kV} / \mathrm{cm}$ ).

\section{CONCLUSIONS}

In the present work, we corroborate that the translationrotation hydrodynamic coupling generate a relatively intense positive component in the stationary electric dichroism of bent rod DNA fragments. Although this positive component is very important for low electric field because it explains dichroism sign, its contribution is meaningful for a wide range of electric field strengths. Results show that the hydrodynamic coupling effect on the electric dichroism is higher when the fragments' symmetry diminishes. Some of the results obtained here agree with those reported in the bibliography. However, there is a difference between the present work and the already existing ones. The latter can be classified in two groups that differ in the study techniques. On the one hand, there are the ones that apply Brownian dynamic simulation and on the other hand there are the ones that apply theoretical methods whose results are applicable in the limit of low electric field. With respect to the first group, the difference lies in the use of completely different study techniques, theory, and computational simulation, while with respect to the second group, it agrees with the use of theoretical methods but it is applicable to higher electric fields.

The calculation procedure for RELD proposed here requires a little computational time. Thus, in the cases of DNA fragments short enough to be considered as rigid particles, the 
mentioned procedure is a useful tool to determine molecular parameters fitting experimental data of RELD as a function of electric field strength.

\section{ACKNOWLEDGMENTS}

Juan P. Umazano is a fellow of Consejo Nacional de Investigaciones Científicas y Técnicas (CONICET) and wishes to thank this institution. He also wishes to thank Professor Dr. J. R. Grigera for contributing to his formation as a researcher.

${ }^{1}$ H. Brenner, Chem. Eng. Sci. 19, 599 (1964).

${ }^{2}$ W. A. Wegener, R. M. Dowben, and V. J. Koester, J. Chem. Phys. 70, 622 (1979).

${ }^{3}$ W. A. Wegener, J. Chem. Phys. 84, 5989 (1986).

${ }^{4}$ W. A. Wegener, J. Chem. Phys. 84, 6005 (1986).

${ }^{5}$ Y. P. Kalmykov, J. Chem. Phys. 131, 074107 (2009).
${ }^{6}$ J. A. Bertolotto, G. B. Roston, M. E. Ascheri, and M. G. Campo, Physica A 327, 185 (2003)

${ }^{7}$ J. A. Bertolotto, G. B. Roston, and M. E. Ascheri, Progr. Colloid Polym. Sci. 128, 25 (2004).

${ }^{8}$ J. A. Bertolotto, G. B. Roston, G. M. Corral, and M. E. Ascheri, AIP Conf. Proc. 913, 151 (2007).

${ }^{9}$ J. A. Bertolotto, Physica A 373, 29 (2007).

${ }^{10}$ J. A. Bertolotto, G. M. Corral, E. M. Farias de La Torre, G. B. Roston, J. Phys.: Condens. Matter. 22, 494101 (2010).

${ }^{11}$ D. Porschke, Biophys. Chem. 49, 127 (1994).

${ }^{12}$ D. Porschke and J. M. Antosiewicz, J. Phys. Chem. B 109, 1034 (2005).

${ }^{13}$ J. M. Antosiewicz and D. Porschke, J. Phys. Chem. B 113, 13988 (2009).

${ }^{14}$ L. Collatz, The Numerical Treatment of Differential Equations (SpringerVerlag, Berlin, 1966).

${ }^{15} \mathrm{H}$. Risken, The Fokker-Planck Equation (Springer-Verlag, Berlin, 1989).

${ }^{16}$ J. A. Schellman, Chem. Rev. 75, 323 (1975).

${ }^{17}$ J. García de la Torre, G. del Rio, and A. Ortega, J. Phys. Chem. B 111, 955 (2007).

${ }^{18}$ J. Antosiewicz and D. Porschke, J. Biomol. Struct. Dyn. 5, 819 (1988).

${ }^{19}$ C. J. F. Böttcher, Theory of Electric Polarization (Elsevier, Amsterdam, 1973). 